\title{
EPIGENETICS
}

\section{Prognosis based on commitment signature}

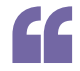

a low epigenetic score was significantly associated with improved overall survival
Several enzymes that regulate DNA cytosine methylation are disrupted in leukaemia. Boris Bartholdy, Maximilian Christopeit and colleagues examined DNA cytosine methylation during physiological haematopoietic stem cell (HSC) differentiation and identified an epigenetic stem cell commitment signature that was prognostic for overall survival in patients with acute myeloid leukaemia (AML).

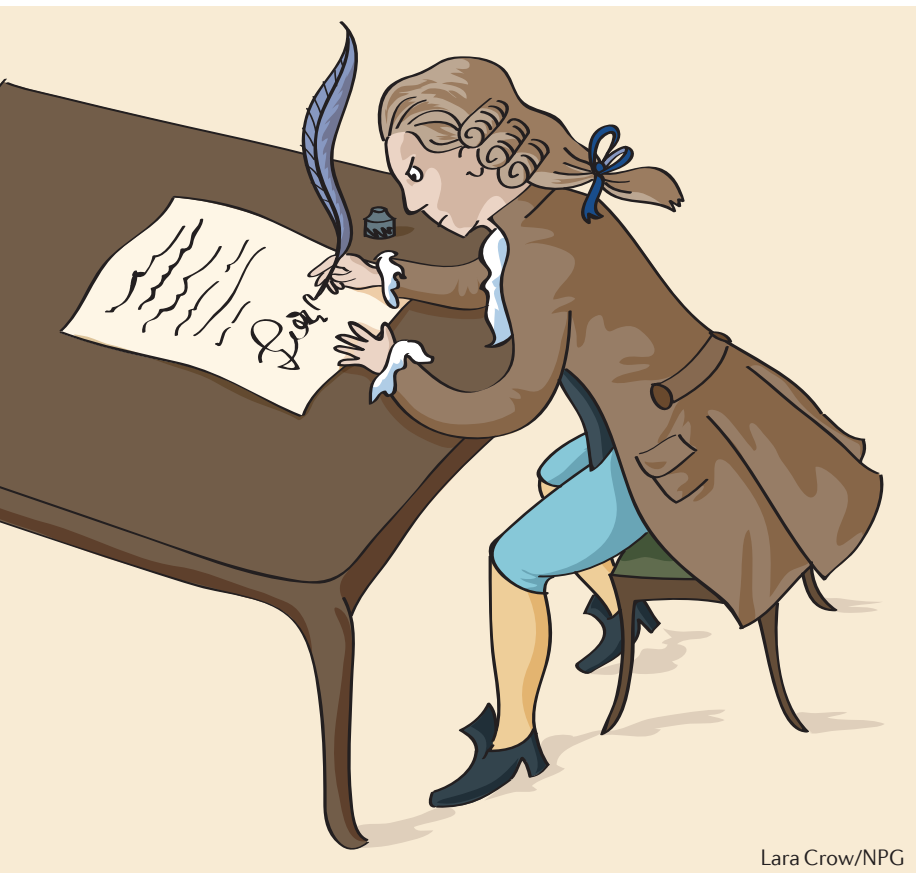

The authors examined the DNA cytosine methylation signature in healthy individuals, starting with purified long-term HSC (LTHSC) and short-term HSC (STHSC) populations, and progressing through to common myeloid progenitor (CMP) and megakaryocyte-erythrocyte progenitor (MEP) populations. The authors found a significant reduction in the median levels of DNA cytosine methylation at the STHSC to CMP commitment step. Analysis of changes at the level of individual loci showed that between the progression of LTHSC to STHSC, $40 \%$ of the 509 significantly differentially methylated loci were demethylated and $60 \%$ were more methylated in the STHSC cells. Similarly, transition from CMP to MEP identified 127 loci (52\%) with decreased methylation levels and 116 (48\%) with increased methylation levels, which indicates predominantly balanced changes in the numbers of hypomethylated and hypermethylated loci. However, the transition between STHSC to CMP showed that, of the 793 differentially methylated loci, 95\% were more methylated in STHSCs than in CMPs. By examining the loci with the most prominent methylation changes across the differentiation stages, the authors identified a 561 loci signature that could distinguish between the differentiation stages - most notably, between the STHSC to CMP transition, in which 516 of the 561 loci were demethylated in the CMP population. This epigenetic signature included methylation changes in genes (both proteincoding and non-coding genes) that are involved in HSC regulation and implicated in leukaemogenesis.

The authors then used this epigenetic signature to analyse published gene expression data and clinical outcome data from three independent cohorts of patients with AML. They found that a low epigenetic score was significantly associated with improved overall survival in the 688-patient data samples that were analysed. Interestingly, mutations in genes that are known to regulate DNA cytosine methylation, such as isocitrate dehydrogenase 1 (IDH1), IDH 2 and methylcytosine dioxygenase 2 (TET2), were not enriched in either the high-scoring or the low-scoring patients. Moreover, the comparison of the epigenetic signature with a previously identified gene expression signature that is prognostic for AML outcome indicated that the epigenetic signature was a more robust indicator of overall survival.

These data indicate that assessing the status of HSC commitment using an epigenetic signature is a valid independent classifier of overall survival in patients with AML.

Nicola McCarthy

ORIGINAL RESEARCH PAPER Bartholdy, B. et al. HSC commitment-associated epigenetic signature is prognostic in acute myeloid leukemia. J.Clin. Invest. http://dx.doi.org/10.1172//Cl71264 (2014) 